\title{
A Proposed Model of Hybrid Cloud Computing to Enhance E-Commerce Services
}

\author{
A. M. Riad \\ Professor of Information System Dept. \\ Faculty of Computer and Information Sciences \\ Mansoura University
}

\author{
E. A. Borhamy \\ Department of Computers and Inf. Systems, Sadat \\ Academy, Cairo Egypt
}

\begin{abstract}
Recent years have witnessed a remarkable success of cloud computing services and applications, while still cost-effective is considered. Recent trends in e-commerce models and applications have employed effectively the benefits of cloud computing. The main focus of many recent types of research has been given to propose models and applications to enhance e-commerce services using cloud computing.

In this paper, the main focus is centered on proposing an effective approach that combines both perspectives of cloud computing parties in an e-commerce environment. A proposed model has been developed for e-commerce as a prototype in hosted to cloud computing. Such a prototype is used to evaluate, test and validate the proposed model based on combining e-commerce to cloud computing. The developed prototype contains e-commerce consumer, service provider, and platform provider (using Microsoft Azure) while the developed website has supported man e-commerce services including orders, shopping, customers and payment services. Results and testing have validated the enhancement gained by e-commerce services while maintaining a cost-effective criteria due to the use of cloud computing.
\end{abstract}

\section{General Terms}

Cloud computing, Information Systems.

\section{Keywords}

Cloud computing, E-commerce, Hybrid, Window Azure.

\section{INTRODUCTION}

Cloud computing is, in fact, the realization of combining many existing technologies (Service Oriented Architecture (SOA), Grid Computing (GC), utility computing, virtualization, and autonomic computing) with new ideas to create efficient and more effective IT solutions [1]. Ravi, 2010, suggested in a report on cloud computing that: enterprises are moving beyond experimentation. They were beginning to develop management software to deal with scaled cloud environment and enterprise level policies for dealing with public and hybrid clouds.

E-commerce came into existence since the late 1970s. Ecommerce is a way of exchanging services and products between consumer and the company through the internet. According to [2], the technical architecture of e-commerce includes hardware and software.

The technical architecture is the base of e-commerce which must maintain its security for the online services and products. Cloud computing makes a substantial impact on the technical architecture of e-commerce, [2]. Piotrowicz and Cuthbertson, 2014 [3], stated that the best e-commerce service on the cloud computing must fulfill the following criteria (to improve the cross-sell and upsell process and decision making and to decrease the operating costs), through an integrated platform and providing customers alternative ways.

The main problem of this research is centered on the challenge of devising an effective approach that combines both perspectives of cloud computing parties in an ecommerce environment.

The goal of this paper is to propose an efficient model using cloud computing to improve e-commerce services outcomes.

This paper is carried out as follows. The second section defines basic concepts, recent trends and challenges of ecommerce and cloud computing and the benefits of hybridizing them. The third section explains the proposed model of Hybrid cloud computing for e-commerce services. The fourth section introduces the implementation of a website for e-commerce services and using business intelligence (BI). The last section concludes the paper.

\section{CLOUD COMPUTING FOR E- COMMERCE}

This section defines basic concepts, recent trends and challenges of e-commerce and cloud computing and the benefits of hybridizing them. E-commerce involves digitally enabled commercial transactions between and among organizations and individuals that occur over the internet and the web [4]. E-commerce activities in cloud computing are carried out by customers; banks; e-commerce companies and cloud service providers [5].

Cloud computing is a type of parallel and distributed system consisting of a collection of inter-connected and virtualized computers that are dynamically provisioned and presented as one or more unified computing resources based on service- 


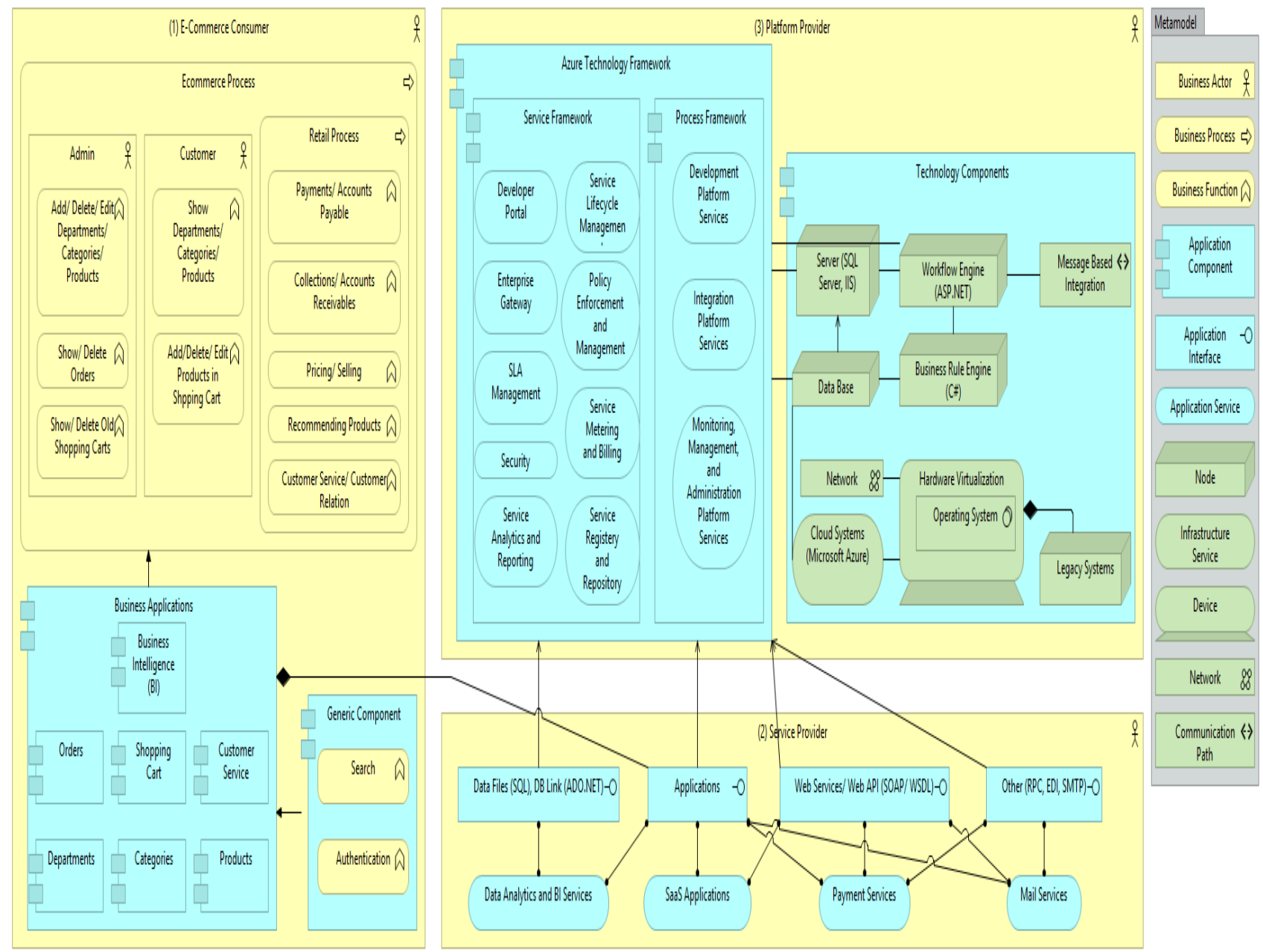

Fig. 1: E-Commerce System Services Architecture.

level agreements established through negotiation between the service provider and consumers [6].

Cloud computing refers to both the applications delivered as services over the internet and the hardware and systems software in the data centers that provide those service. Cloud computing is composed of three layers, Software as a Service (SaaS), Platform as a Service (PaaS) and Infrastructure as a Service (IaaS) [7].

The most important benefit of the cloud computing is its ability to help businesses and organizations to increase their productivity and reduce their cost of operations and maintenance while providing new products to their customers, [8]. Cloud computing can also benefit organizations, industries, and economies by providing companies' ways in which they can develop new products and offer new services to its clients [9].

The cloud computing for e-commerce has several benefits [10]. These benefits are the trust and security of cloud computing, investments tailored to the needs of e-commerce, cloud computing scalability and flexibility, mobility, global expansion, cost of construction and operations, quality of ecommerce

There are three different ways to deploy cloud computing resources: public cloud, private cloud, and hybrid cloud. Public clouds are owned and operated by a third-party cloud sources used exclusively by a single business or organization, physically located on the company's on-site datacenter. Hybrid clouds combine public and private clouds, bound together by technology that allows data and applications to be shared between them that gives businesses greater flexibility and more deployment options [11].

\section{PROPOSED ARCHITECTURE OF E-COMMERCE SYSTEM SERVICES}

This section explains the proposed model of Hybrid cloud computing for e-commerce services. Figure 1, illustrates the view of the e-commerce system services architecture including a meta-model of the relevant concepts in the top right. The service platform has three components, namely Ecommerce consumer, Service provider and Platform provider [12]. The rest of this section explains in detail the platform components.

\subsection{E-commerce consumer}

It includes service consumer and actor selling goods partially or exclusively over the online channel. There are seven business applications related to departments in the company, categories in the departments, products in the categories, orders of products, shopping cart that contains orders, business intelligence application to recommend products and customer service to deal with customer inquiries. Also, there's 
a generic component that has the general search function and authentication for users of the e-commerce system.

The e-commerce process contains two types of actors. The administrator who is able to add, delete or edit the departments, categories or products in the system. Also, he can show or delete orders, and show or delete old shopping carts. The other actor is the customer who can show departments, categories or products, and they can add, delete or edit the products in the shopping cart. The retail process handles the accounts of customers that have ordered but haven't paid yet and also handle the accounts that paid for the orders. Also, the process handles the selling and pricing and offers on the products. Also, the process includes the inquiries of customers and uses the business intelligence application for recommended products for the customer.

\subsection{Service Provider}

The service provider can either issue pure IT services or be a supply chain partner that provides business services (B2B ecommerce). Both service types have to be integrated into information system level for seamless process execution. However, a more important aspect is to obtain a comprehensive picture of potential service interfaces the platform needs to support. Four different services interfaces have been identified as follows:

\subsubsection{Web services}

Message-based integration can be realized through modern web services or web APIs that communicate over HTTP and can be consumed with state of the art integration tools and techniques. This kind of interface is suitable for standalone services such as payment services and to access resources of SaaS applications and in a programmable manner.

\subsubsection{Communication protocols}

Another interface type is based on more specialized protocols that can be considered as an older technique to integrate services. Despite their higher complexity and technical dependencies, those protocols are still widely used to integrate legacy systems or communication services such as mail.

\subsubsection{Applications}

Web applications are generally used as user interfaces in SaaS as well as in analytical services and reporting in the form of dashboards.

\subsubsection{Data files}

On the backend analytical, services will be integrated through an interface type that allows the exchange of large amounts of data. Instead, the integration will rather be based on data extraction, transfer, and loading or through database links like ADO.NET that connect with SQL data files.

\subsection{Platform provider (Microsoft Azure)}

Microsoft Azure is used as a platform of e-commerce system based on the cloud that has multiple virtual machines to virtualize client requests and admin usage of the system. It includes all legacy systems required to provide the system and also provide the network between different actors.

The cloud framework (Azure support service framework) services are shown in Table 1 For the company in order to process and integrate these services, the process framework is explained in Table 2 [12]. Technology components are SQL to deal with data, SQL Server and IIS as servers, ASP.NET to deal with the workflow and C\# for decoding the BI.

\section{IMPLEMENTING PROPOSED}

Table 1. Cloud Framework Services

\begin{tabular}{|c|c|}
\hline $\begin{array}{c}\text { Service } \\
\text { Framework }\end{array}$ & Features \\
\hline Developer portal: & $\begin{array}{l}\text { In the developer portal, companies } \\
\text { should provide relevant and } \\
\text { comprehensive aspects of their APIs } \\
\text { such as API documentation, policy, } \\
\text { terms, and agreement, testing } \\
\text { environment (sandbox or real), or } \\
\text { API versioning. }\end{array}$ \\
\hline $\begin{array}{l}\text { Enterprise } \\
\text { gateway: }\end{array}$ & $\begin{array}{l}\text { Management of the interaction } \\
\text { between the API and external API } \\
\text { consumers. }\end{array}$ \\
\hline $\begin{array}{l}\text { Policy enforcement } \\
\text { and management: }\end{array}$ & $\begin{array}{l}\text { Management of both, design time and } \\
\text { runtime policies of services. Design } \\
\text { time policies are concerned with } \\
\text { aspects such as design guidelines or } \\
\text { security mechanism while run-time } \\
\text { policies are concerned with the } \\
\text { operational environment and } \\
\text { requirements that have to be met by } \\
\text { the service at runtime. }\end{array}$ \\
\hline Security: & $\begin{array}{l}\text { The difference between security in } \\
\text { SOA governance and API } \\
\text { management is that in SOA } \\
\text { governance, the organization } \\
\text { administers internal and known users } \\
\text { while API management handles } \\
\text { external and unknown users. API } \\
\text { security manages additional aspects } \\
\text { like authorization and authentication, } \\
\text { API key management, as well as } \\
\text { identity and credential management. }\end{array}$ \\
\hline $\begin{array}{l}\text { Service analytics } \\
\text { and reporting: }\end{array}$ & $\begin{array}{l}\text { Exploration of insightful traffic } \\
\text { analytics and reports of API activities } \\
\text { with respect to developers account, } \\
\text { application, or services as well as } \\
\text { observation of the overall API usage } \\
\text { and trends. }\end{array}$ \\
\hline $\begin{array}{l}\text { Service level } \\
\text { agreement: }\end{array}$ & $\begin{array}{l}\text { Management of service levels as } \\
\text { stated in SLA contract, service } \\
\text { evaluation as well as fees for } \\
\text { consuming the service and fines in } \\
\text { case of contract violation }\end{array}$ \\
\hline $\begin{array}{l}\text { Service lifecycle } \\
\text { management: }\end{array}$ & $\begin{array}{l}\text { Managing the design, development, } \\
\text { and delivery of individual services in } \\
\text { an SOA. The tasks include change } \\
\text { management procedure, service } \\
\text { registration and even deciding on } \\
\text { service granularity. }\end{array}$ \\
\hline $\begin{array}{l}\text { Service metering } \\
\text { and billing: }\end{array}$ & $\begin{array}{l}\text { Monitor and measure service usage as } \\
\text { the basis for billing and calculation } \\
\text { for the service consumers. Also, the } \\
\text { service performance can be } \\
\text { monitored regularly. }\end{array}$ \\
\hline $\begin{array}{l}\text { Service registry } \\
\text { and repository: }\end{array}$ & $\begin{array}{l}\text { The catalog of services and } \\
\text { management of their publication. } \\
\text { Definition of taxonomies of the } \\
\text { published services allowing } \\
\text { consumers to find suitable services to } \\
\text { their needs. While the service registry } \\
\text { only contains service references, the } \\
\text { service repository is the actual holder } \\
\text { of documentation, policies, and } \\
\text { metadata about the versioning of the } \\
\text { service. }\end{array}$ \\
\hline
\end{tabular}




\section{FRAMEWORK}

This section introduces the implementation of a website for ecommerce services and how we integrated payment services using PayPal in it and how we implemented product recommendation by the use of business intelligence.

\subsection{Implementing Cloud E-Commerce Site}

To implement the e-commerce services with windows azure as a platform; creates a cloud e-commerce web site with several servers [13]. Implementing process needs three phases: getting a site up, creating your own shopping cart and processing orders.

\subsubsection{Getting a site up}

Starting by putting together the basic site architecture, deciding how the different parts of the application will work together. The product catalog is built into this architecture to achieve the following:-

- Design a database for storing a product catalog containing categories, subcategories, and products.

- Write the SQL code and C\# code for accessing that data.

- Build an attractive and user interface (UI) that allows for easy catalog browsing.

- Implement an efficient error-reporting system that notifies the administrator in case the site runs into trouble and displays a message to the visitor when a critical error occurs.

- Integrate an external payment processor (with examples for PayPal) to allow visitors to order your products.

- Provide a free-text search engine for the database.

\subsubsection{Creating your own shopping cart}

Creating your own shopping cart using PayPal's shopping cart you can store complete orders in your database as part of the order process, and then use that data to learn about your customers. With additional work, you also can use the shopping basket and checkout as a platform for selling more products. At the end of this phase, your site will be fully operational. Any extended features can be added within the existing PayPal-based payment system. Also, processing orders and using credit cards and check site's security can be performed [14].

\subsubsection{Processing orders}

The core of e-commerce is handling orders and credit cards. PayPal is used for the following reasons:

- Cost: PayPal is not expensive.

- Freedom: PayPal has a fairly strict set of terms and conditions.

- Integration: you can integrate your store and your warehouse to whatever extent you require.

- Information: you can record and collect all the information involved in the transaction.

By the end of phase III, you will have an e-commerce site with a searchable product catalog, shopping cart, secure checkout, and complete order-processing system.

\subsection{Receiving Payments Using Paypal}

In the first stage of development, you need to integrate the shopping cart and checkout functionality from PayPal. In the second stage of development, after you create your own shopping cart, you'll only need to rely on PayPal's checkout mechanism. To accept payments, you need to add two important elements to the user interface part of the site: add to cart buttons for each product and a view cart button somewhere on the page. PayPal makes adding these buttons a piece of cake. The functionality of those buttons is performed by secure links to the PayPal website.

Figure 2 shows the PayPal shopping cart in action. This figure contains 4 buttons:

- Delete: To delete an item from the cart

- Update quantities: To Change the quantity purchased

- Proceed to checkout: To Go to PayPal to make the order

- Continue shopping: to go back to the site and purchase more items

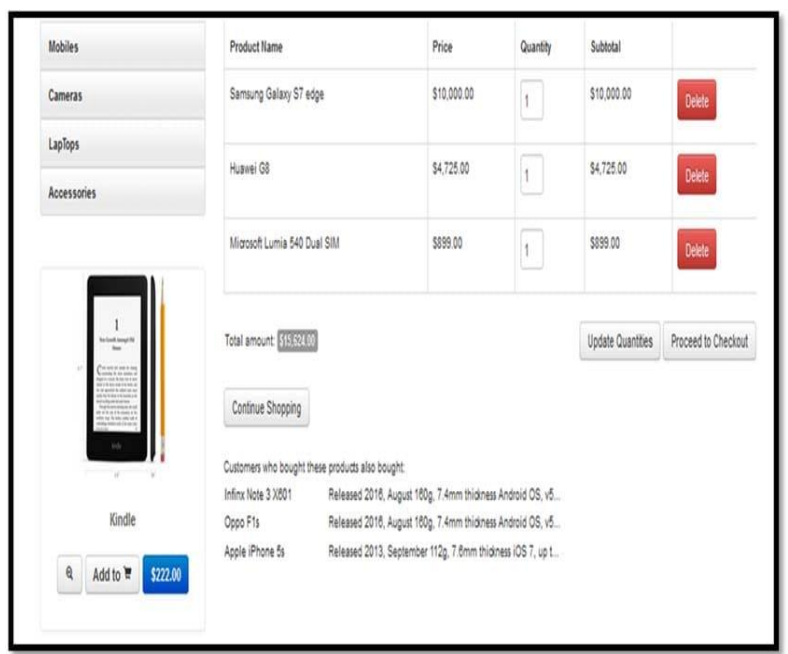

Fig. 2: PayPal shopping cart in action

While Figure 3 shows the Using of the PayPal single item purchases feature.

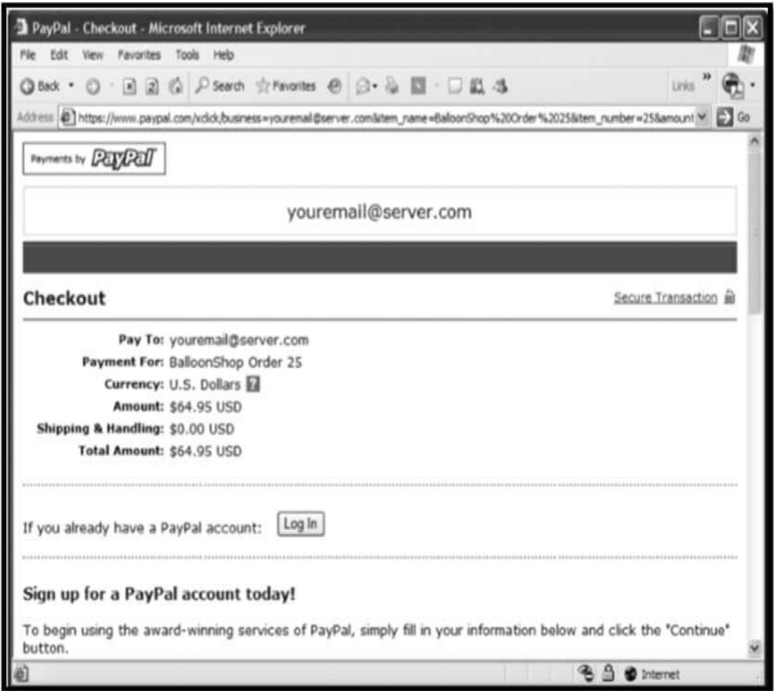

Fig. 3: Using the PayPal single item purchases feature. 


\subsection{Using Business Intelligent In Product Recommendations}

In the electronics shop, you will implement the dynamic recommendations system in the visitor's shopping cart and in the product details page. After adding the new bits to your shop, the shopping cart page will contain the product recommendations list at the bottom of the page as shown in Figure 4 .

What the logic of recommending products that were ordered together with another specific product. Afterward, the recommendations for the shopping cart page will function in a similar way but will take more products into consideration. So you need to find out what other products were bought by customers who also bought the product for which you are calculating the recommendations.

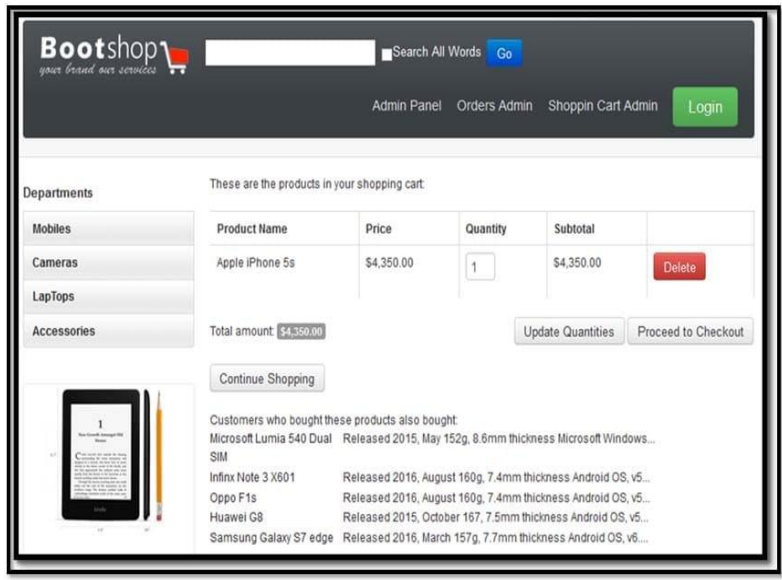

Fig. 5: Product recommendations.

Creating the user interface for product recommendations implies three major steps:

- Creating a new web user control that displays the product recommendations. This new control will be named product recommendations.ascx.

- Adding product recommendations.ascx to the product.aspx, where it must display the "customers who bought this product also bought:" list.

- Adding product recommendations.ascx to the shopping cart.aspx, where it displays the "customers who bought these products also bought:" list [15].

\section{CONCLUSIONS}

The study proposed an architecture of e-commerce system services that contain e-commerce consumer, service provider, and platform provider.

The e-commerce consumer contains two types of actors. The administrator who is able to add, delete or edit the

\section{REFERENCES}

[1] B Dustin, A. and Quint, S. 2010. Cloud computing for the enterprise: part 1: capturing the cloud. Understanding cloud computing and related technologies. IBM. departments, categories or products in the system. Also, he can show or delete orders, and show or delete old shopping carts. The other actor is the customer who can show departments, categories or products, and they can add, delete or edit the products in the shopping cart. The retail process handles the accounts of customers that have ordered but haven't paid yet and also handle the accounts that paid for the orders. Also, the process handles the selling and pricing and offers on the products. Also, the process handles the inquiries of customers and uses the business intelligent application in order to make recommended products for the customer

The service provider can either issue pure IT services or be a supply chain partner that provides business services (B2B ecommerce). The service provider provides web services, communication protocols, applications, and data files.

Microsoft Azure is the platform provider which is the cloud system used in the e-commerce system that has multiple virtual machines to virtualize client requests and admin usage of the system. It includes all legacy systems required to provide the system and also provide the network between different actors in the system.

The study implemented cloud e-commerce website that has the services of orders, shopping, customer service, and the payments services. The tools and technologies used in the website include windows azure, ASP.NET, and SQL server. The study discussed in details the phases of implementing the cloud website and the different services that include order, products, customer, etc.

The study used business intelligence in implementing the payment services that used PayPal that contributed in product recommendation. In the website, you will notice the dynamic recommendations system in the visitor's shopping cart and in the product details. After adding the new bits to your shop, the shopping cart page will contain the product recommendations list at the bottom of the page. The logic of recommending products that you need to find out what other products were bought by customers who also bought the product for which you're calculating the recommendations.

Table 2: Process framework Features.

\begin{tabular}{|c|l|}
\hline $\begin{array}{c}\text { Process } \\
\text { Framework }\end{array}$ & \multicolumn{1}{|c|}{ Features } \\
$\begin{array}{c}\text { Development } \\
\text { platform services: }\end{array}$ & $\begin{array}{l}\text { Manages service integration process } \\
\text { flows throughout their lifecycle } \\
\text { including modeling, development, } \\
\text { configuration, testing, and } \\
\text { deployment. }\end{array}$ \\
\hline $\begin{array}{l}\text { Consists of aspects that ensure } \\
\text { seamless integration flow both at } \\
\text { design time (service orchestration) }\end{array}$ \\
and runtime (process execution). \\
Integration & $\begin{array}{l}\text { These aspects include but are not } \\
\text { limited to message transformation } \\
\text { and routing, an Integrated }\end{array}$ \\
platform services: & $\begin{array}{l}\text { Development Environment (IDE), } \\
\text { adapters, flow management, protocol } \\
\text { conversion, service virtualization, and } \\
\text { security federation. }\end{array}$ \\
Monitoring, & $\begin{array}{l}\text { Takes care of deployment and } \\
\text { administration of integration flows, } \\
\text { monitor their execution and manage } \\
\text { their behavior. Covers several aspects }\end{array}$ \\
such as technical and business \\
activity monitoring, logging and \\
administration \\
platform services:
\end{tabular}


[2] Kenneth, C.L. and GUERCIO, C., 2017. E-commerce: business, technology, society.

[3] Piotrowicz, W. and Cuthbertson, R., 2014. Introduction to the special issue information technology in retail: Toward omnichannel retailing. International Journal of Electronic Commerce, 18(4), pp.5-16.

[4] Turban, E., Outland, J., King, D., Lee, J.K., Liang, T.P. and Turban, D.C., 2017. Electronic commerce 2018: a managerial and social networks perspective. Springer.

[5] Wang, D., 2013. Influences of cloud computing on ecommerce businesses and industry. Journal of software Engineering and Applications, 6(06), p.313.

[6] Meng, K. 2008. A walk in the cloud: uncovering cloud computing. China Network World, 12-14.

[7] Craig-Wood, K. 2010. IaaS vs. PaaS vs. SaaS definitions. Kates Comment.

[8] Ko, R., \& Choo, R. 2015. The Cloud Security Ecosystem: Technical, Legal, Business and Management Issues. Syngress.

[9] Joshi, J. M., \& Dumbre, G. M. (2017). Basic Concept of ECommerce. International Research Journal of Multidisciplinary Studies, 3(3).
[10] Opara-Martins, J., Sahandi, R., \& Tian, F. 2016. Critical analysis of vendor lock-in and its impact on cloud computing migration: a business perspective. Journal of Cloud Computing, 5(1), 4.

[11] Carter, P. A. 2015. SQL Data Files in Windows Azure In Pro SQL Server Administration (pp. 945-959). Apress, Berkeley, CA.

[12] Aulkemeier, F., Paramartha, M. A., Iacob, M. E., \& van Hillegersberg, J. 2016. A pluggable service platform architecture for e-commerce. Information systems and ebusiness management, 14(3), 469-489.

[13] Klein, C., Maggio, M., Årzén, K. E., \& HernándezRodriguez, F. (2014, May). Brownout: Building more robust cloud applications. In Proceedings of the 36th International Conference on Software Engineering (pp. 700-711). ACM.

[14] Ouf, S., \& Nasr, M. 2015. Cloud computing: the future of big data management. International Journal of Cloud Applications and Computing (IJCAC), 5(2), 53-61.

[15] Lu, J., Wu, D., Mao, M., Wang, W., \& Zhang, G. 2015. Recommender system application developments: a survey. Decision Support Systems, 74, 12-32. 